\title{
Ácaros plumícolas (Acari: Astigmata) em aves do Cerrado do Distrito Federal, Brasil
}

\author{
Mieko Ferreira Kanegae ${ }^{1,4}$; Michel Valim ${ }^{2}$; Marcelo Andrade da Fonseca ${ }^{2}$; \\ Miguel Ângelo Marini ${ }^{3}$; Nicolau Maués Serra Freire ${ }^{2}$ \\ ${ }^{I}$ Pós-graduação em Ecologia, Departamento de Ecologia, Instituto de Biociências, \\ Universidade de São Paulo - USP \\ Rua do Matão, 321, Travessa 14, CEP 05508-900 São Paulo, SP, Brasil \\ ${ }^{2}$ Laboratório de Ixodides, Departamento de Entomologia, \\ Instituto Oswaldo Cruz - FIOCRUZ, \\ Av. Brasil, 4365, CEP 21040-900, Manguinhos, Rio de Janeiro, RJ, Brasil \\ ${ }^{3}$ Departamento de Zoologia, IB, Instituto de Ciências Biológicas, \\ Universidade de Brasília - UnB, \\ Asa Norte, CEP 70910-900, Brasilia, DF, Brasil \\ ${ }^{4}$ Autor para correspondência: Mieko Ferreira Kanegae, e-mail: miekok@terra.com.br
}

Kanegae, M. F.; Valim, M. P.; Fonseca, M. A.; Marini, M. A.; Freire, N. M. S. Feather mites (Acari: Astigmata) on birds of Cerrado in Distrito Federal, Brazil. Biota Neotrop., vol. 8, no. 1, Jan./Mar. 2008. Available from: <http://www.biotaneotropica.org.br/v8n1/pt/abstract?article+bn00208012008>.

\begin{abstract}
The goal of this study was to identify feather mites on Cerrado birds. This study was conducted at Fazenda Água Limpa (FAL), Distrito Federal, Brazil, between January and August 2002. Birds were captured with mist nets and banded. Were sampled 696 birds, belonging to 83 species and 25 bird families of Passeriformes and non-Passeriformes. Feather mites were found on five families: Analgidae, Trouessartiidae, Proctophyllodidae, Avenzoariidae and Psoroptoididae. The present work reports new occurrences of feather mites on captured hosts. The family Proctophyllodidae and the genus Pterodectes were the most prevalent group of feather mites collected. Tyrannidae, Pipridae, Emberizidae and Thraupidae were the bird families with most host numbers, with parasites from three taxa: Trouessartiidae, Proctophyllodidae and Analgidae. Considering the birds captured the prevalence was $44.98 \%$. Trochilidae, Conopophagidae, Furnariidae, Tityridae, Turdidae, Mimidae, Cardinalidae, and Parulidae were the families with major feather mites prevalence $(\geq 50 \%)$.
\end{abstract}

Keywords: biodiversity, feather mites, prevalence, community, Cerrado.

Kanegae, M. F.; Valim, M. P.; Fonseca, M. A.; Marini, M. A.; Freire, N. M. S. Ácaros plumícolas (Acari: Astigmata) em aves do Cerrado do Distrito Federal, Brasil. Biota Neotrop., vol. 8, no. 1, jan./mar. 2008. Disponível em: <http://www.biotaneotropica.org.br/v8n1/pt/abstract?article+bn00208012008>.

Resumo: O objetivo do estudo foi identificar os ácaros plumícolas em aves do cerrado. O mesmo foi realizado na Fazenda Água Limpa (FAL), Distrito Federal, Brasil, entre janeiro e agosto de 2002. As aves foram capturadas com rede de neblina e anilhadas. Foram amostradas 696 aves pertencentes a 83 espécies e 25 famílias de Passeriformes e não-Passeriformes. Foram encontrados ácaros plumícolas pertencentes a cinco famílias: Analgidae, Trouessartiidae, Proctophyllodidae, Avenzoariidae e Psoroptoididae. O presente trabalho contribui com novos registros de ácaros plumícolas nos hospedeiros capturados. A família Proctophyllodidae e o gênero Pterodectes foram os mais prevalentes grupos de ácaros plumícolas coletados. Considerando todas as aves capturadas, a prevalência foi de $44,98 \%$. As maiores prevalências (>50\%) de ácaros plumícolas foram encontradas para as famílias Trochilidae, Conopophagidae, Furnariidae, Tityridae, Turdidae, Mimidae, Cardinalidae e Parulidae.

Palavras-chave: biodiversidade, ácaros plumícolas, prevalência, comunidade, Cerrado. 


\section{Introdução}

Os ácaros plumícolas compõem o grupo mais diverso e numeroso de artrópodes associado às aves. Contudo, a natureza de sua interação com o hospedeiro continua incerta (comensalismo, mutualismo ou parasitismo) (Figuerola 2000, Blanco et al. 2001). Esses ácaros pertencem a subordem Astigmata e as superfamílias Analgoidea, Pterolichoidea e Freyanoidea (Gaud \& Atyeo 1996), sendo apenas a primeira encontrada em aves Passeriformes. Existem aproximadamente 2.400 espécies de ácaros plumícolas conhecidas (Mironov 2003), porém apenas dois gêneros, Dubininia Gaud \& Mouchet, 1959 e Megninia Berlese, 1883, foram relatados prejudicando seus hospedeiros induzindo a deplumação (Proctor \& Owens 2000).

Todos os principais grupos de aves possuem ácaros plumícolas, sendo que cada ordem apresenta uma fauna específica de ácaros (Gaud \& Atyeo 1996). Dentro das ordens a especificidade destes ácaros pode ser relacionada às famílias, gêneros ou até mesmo às espécies de aves (Gaud \& Atyeo 1976). Essa especificidade é consequiência do mecanismo de transmissão que ocorre principalmente através do contato físico entre casais, pais com filhotes e entre indivíduos de bandos (Gaud \& Atyeo 1996, Proctor \& Owens 2000, Proctor 2003).

Os primeiros trabalhos publicados no Brasil com ácaros plumícolas foram feitos por Herbert F. Berla em meados do século passado (Pacheco \& Parrini 1999), exclusivamente com enfoque taxonômico. Há poucos estudos com relação à identificação desses ácaros em aves brasileiras, incluindo os realizados em áreas de florestas e cerrado de Minas Gerais (Rojas 1998), nas aves da zona da Mata Norte de Pernambuco (Roda \& Farias 1999), em aves da Floresta Atlântica (Lyra-Neves et al. 2000, 2003), em hospedeiros específicos como o Ramphocelus carbo (Pallas, 1764) presente na várzea do rio Guamá, Pará (Carvalho \& Serra-Freire 2001) e o Turdus albicollis Vieillot, 1818 na Mata Atlântica da Ilha Grande, Rio de Janeiro (Storni et al. 2005). Recentemente estudos vem sendo conduzidos com a descrição de novas espécies e a redescrições de ácaros plumícolas em aves neotropicais (e.g. Hernandes \& Valim 2005, 2006, OConnor et al. 2005, Valim \& Hernandes 2006), o que mostra ainda um grande desconhecimento para fauna de ácaros nessa região geográfica.

O presente trabalho teve como objetivo identificar os ácaros plumícolas, ao menor táxon possível, de aves capturadas em região de cerrado no Distrito Federal, Brasil.

\section{Material e Métodos}

O trabalho foi realizado na Fazenda Água Limpa (FAL) localizada $20 \mathrm{~km}$ a sudeste de Brasília, Distrito Federal (15 $57^{\prime} \mathrm{S}$ and $47^{\circ} 56^{\prime}$ W). A FAL está inserida na área de proteção ambiental das Bacias do Gama e Cabeça de Veado, possuindo uma área de 4.340 ha destinada principalmente à preservação, com áreas de produção agrícola e pastoril (Ratter 1980). O estudo foi desenvolvido em dois ambientes de Cerrado: floresta (mata de galeria do Capetinga) e cerrado (campo sujo e cerrado sensu stricto).

O Cerrado representa a maior formação savânica da América do Sul, ocupando uma área de 1,8 milhões de $\mathrm{km}^{2}$ (23\% do território brasileiro) (Ab'Saber 1983). As altitudes variam de $500 \mathrm{~m}$ a mais de 1.700 m (Eiten 1972). Possui clima sazonal com duas estações bem definidas: invernos secos (abril-setembro) e verões chuvosos (outubro-março) com temperaturas variando entre 17 a $22^{\circ} \mathrm{C}$ (Klink \& Machado 2005).

Os dados foram coletados de janeiro a agosto de 2002, perfazendo uma média de 8 dias/mês de campo. A captura das aves foi realizada com 15 redes ornitológicas de $12 \times 2,5 \mathrm{~m}$, abertas nas primeiras horas da manhã (6:00-7:00 horas) e fechadas às 13:00 horas. As aves foram colocadas em sacos individuais com o tecido poroso para posterior marcação com anilhas metálicas cedidas pelo Centro Nacional de
Pesquisa para Conservação das Aves Silvestres / Instituto Brasileiro do Meio Ambiente e dos Recursos Naturais Renováveis (CEMAVE/ IBAMA).

Para verificar a presença de ácaros plumícolas foi realizada uma inspeção das penas do corpo, das asas e cauda contra a luz do sol. As amostras de ácaros obtidas foram retiradas de diferentes locais das penas, sendo considerada uma amostra aquela retirada em um indivíduo. Os ácaros foram coletados e conservados em etanol 70\%, sendo posteriormente enviados para montagem e identificação no Laboratório de Ixodides no Instituto Oswaldo Cruz / FIOCRUZ (Fundação Oswaldo Cruz). Os ácaros foram montados em lâminas de microscopia usando o meio de Hoyer (Flechtmann 1975).

A classificação das aves seguiu o sugerido pelo Comitê Brasileiro de Registros Ornitológicos (2005); a dos ácaros plumícolas seguiu Gaud \& Atyeo (1996) para os táxons supragenéricos e Atyeo \& Braasch (1966), Atyeo (1966), Orwig (1968), Park \& Atyeo (1971a, 1973, 1974, 1975) e Santana (1976) para as categorias específicas.

É importante ressaltar que devido à metodologia utilizada na coleta dos ácaros sobre as aves, alguns espécimes não foram coletados a contento para diagnose mais apurada. Algumas amostras foram representadas apenas por fêmeas, o que dificulta a identificação genérica (e.g. Proctophyllodidae) (Park \& Atyeo 1971b, Atyeo \& Braasch 1966); ou apenas por ninfas, o que limita a identificação ao nível de superfamília e/ou família (Gaud \& Atyeo 1996).

A prevalência foi calculada seguindo a proposição de Bush et al. (1997), sendo excluídas para esses cálculos as aves recapturadas. Os dados de prevalência não incluíram os espécimes recapturados. Resultados considerados "contaminação" do material durante a sua coleta e/ou processamento (e.g. encontro de um ácaro caracteristicamente de um grupo de aves em outro completamente distinto, representado por poucos exemplares) foram descartados das análises de prevalência, os mesmos são comentados na discussão.

\section{Resultados e Discussão}

Foram capturados 696 indivíduos (80 recapturas), pertencentes a 83 espécies e distribuídas em oito famílias de não-Passeriformes (66 indivíduos) e 17 famílias de Passeriformes (630 indivíduos). Deste total, apenas 277 indivíduos $(44,98 \%)$ pertencentes a 73 espécies $(87,95 \%)$ em 17 famílias $(68,0 \%)$ apresentaram ácaros plumícolas (Tabela 1). Foram encontrados 22 gêneros de ácaros, todos pertencentes à superfamília Analgoidea, distribuídos por cinco famílias: Analgidae (02), Trouessartiidae (04), Proctophyllodidae (12), Avenzoariidae (02) e Psoroptoididae (01). A partir do material analisado apenas foi possível identificar 12 táxons ao nível específico, todos pertencentes às famílias Trouessartiidae e Proctophyllodidae (Tabela 1). É provável que grande parte das espécies que não puderam ser especificamente identificadas sejam novas para ciência e devem ser tratadas em trabalho taxonômico para tal fim.

Foi observada em 65 indivíduos a ocorrência de duas famílias de ácaros: Proctophyllodidae e Trouessartiidae (55); Proctophyllodidae e Analgidae (4); Analgidae e Trouessartiidae (3); Psoroptoididae e Trouessartiidae (1); Trouessartiidae e Avenzoaridae (2). A ocorrência de três famílias de ácaros foi observada em cinco indivíduos: (3) com Analgidae, Trouessartiidae e Proctophyllodidae (Ammodramus humeralis - 2 e Basileuterus flaveolus - 1); e (2) com Psoroptoididae, Trouessartiidae e Proctophyllodidae (Conopophaga lineata e Basileuterus hypoleucus) (Tabela 1).

Analisando separadamente a prevalência de ácaros plumícolas entre as famílias, Trochilidae, Conopophagidae, Furnariidae, Tityridae, Turdidae, Mimidae, Cardinalidae e Parulidae apresentaram prevalências $\geq 50 \%$ (Figura 1). Outras famílias, como Tyrannidae e Emberizidae, tiveram suas prevalências mais baixas, provavelmente 
Tabela 1. Aves e seus respectivos ácaros plumícolas encontrados na Fazenda Água Limpa, Brasília, Distrito Federal, Brasil, durante o período de janeiro a agosto de 2002.

Table 1. Feather mites collected on birds of Fazenda Água Limpa, Brasília, Distrito Federal, Brazil, between January and August, 2002.

\begin{tabular}{|c|c|c|c|c|c|c|c|}
\hline Espécies de aves & $\mathbf{N}$ & $\mathbf{R c}$ & $\operatorname{Prv}(\%)$ & Ácaros plumícolas & $\pi$ & 9 & Imaturos \\
\hline \multicolumn{8}{|l|}{ TINAMIFORMES } \\
\hline Tinamidae (1) & 2 & 0 & - & & & & \\
\hline Crypturellus undulatus (Temminck, 1815) & 2 & 0 & - & - & - & - & - \\
\hline \multicolumn{8}{|l|}{ FALCONIFORMES } \\
\hline Falconidae (1) & 1 & 0 & - & & & & \\
\hline Falco femoralis (Temminck, 1822) & 1 & 0 & - & - & - & - & - \\
\hline \multicolumn{8}{|l|}{ COLUMBIFORMES } \\
\hline Columbidae (3) & 5 & 0 & - & & & & \\
\hline Columbina talpacoti (Temminck, 1811) & 2 & 0 & - & - & - & - & - \\
\hline Leptotila verreauxi (Bonaparte, 1855) & 1 & 0 & - & - & - & - & - \\
\hline Leptotila rufaxilla (Richard \& Bernard, 1792) & 2 & 0 & - & - & - & - & - \\
\hline \multicolumn{8}{|l|}{ APODIFORMES } \\
\hline Trochilidae (5) & 36 & 5 & 69,70 & & & & \\
\hline \multicolumn{8}{|l|}{ Phaethornithinae } \\
\hline Phaethornis pretrei (Lesson \& Delattre, 1839) & 4 & 0 & 25,00 & Proctophyllodidae & 0 & 0 & 2 \\
\hline \multicolumn{8}{|l|}{ Trochilinae } \\
\hline \multirow[t]{3}{*}{ Colibri serrirostris (Vieillot, 1816) } & 7 & 0 & 28,57 & Allodectes amadoi & 1 & 7 & 1 \\
\hline & & & 14,29 & Neocalcealges sp.* & 1 & 0 & 4 \\
\hline & & & 42,86 & Toxerodectes subulatus & 9 & 25 & 21 \\
\hline $\begin{array}{l}\text { Chlorostilbon lucidus (d'Orbigny \& } \\
\text { Lafresnaye, 1838) }\end{array}$ & 5 & 0 & 40,00 & Proctophyllodes huitzilopchtlii & 8 & 27 & 9 \\
\hline \multirow[t]{3}{*}{ Thalurania furcata (Gmelin, 1788) } & 13 & 5 & 12,50 & Allodectes amadoi & 2 & 1 & 10 \\
\hline & & & 87,50 & Toxerodectes biscutatus & 23 & 69 & 13 \\
\hline & & & 12,50 & Pterodectes sp.* & 1 & 0 & 0 \\
\hline Amazilia fimbriata (Gmelin, 1788) & 7 & 0 & 71,43 & Proctophyllodes huitzilopchtlii & 9 & 71 & 9 \\
\hline \multicolumn{8}{|l|}{ CORACIIFORMES } \\
\hline Momotidae (1) & 2 & 0 & - & & & & \\
\hline Baryphthengus ruficapillus (Vieillot, 1818) & 2 & 0 & - & - & - & - & - \\
\hline Galbulidae (1) & 2 & 0 & - & & & & \\
\hline Galbula ruficauda (Cuvier, 1816) & 2 & 0 & - & - & - & - & - \\
\hline Bucconidae (1) & 10 & 1 & - & & & & \\
\hline Nystalus chacuru (Vieillot, 1816) & 10 & 1 & - & - & - & - & - \\
\hline \multicolumn{8}{|l|}{ PICIFORMES } \\
\hline Picidae (3) & 8 & 0 & 50,00 & & & & \\
\hline Picumnus albosquamatus (d'Orbigny, 1840) & 2 & 0 & 50,00 & Pseudalges sp. & 14 & 7 & 2 \\
\hline Veniliornis passerinus (Linnaeus, 1766) & 4 & 0 & 25,00 & Pterotrogus sp. & 7 & 4 & 3 \\
\hline \multirow[t]{2}{*}{ Picoides mixtus (Boddaert, 1783) } & 2 & 0 & 50,00 & Pandalurinae & 12 & 37 & 5 \\
\hline & & & 50,00 & Pterodectes sp. & 19 & 24 & 35 \\
\hline \multicolumn{8}{|l|}{ PASSERIFORMES } \\
\hline Thamnophilidae (2) & 12 & 1 & 54,55 & & & & \\
\hline \multirow[t]{2}{*}{ Thamnophilus punctatus (Shaw, 1809) } & 4 & 1 & 33,33 & Calcealges novimundus & 4 & 6 & 1 \\
\hline & & & 33,33 & Pterodectinae & 0 & 4 & 0 \\
\hline Thamnophilus caerulescens (Vieillot, 1816) & 6 & 0 & 50,00 & Calcealges novimundus & 8 & 13 & 2 \\
\hline \multirow[t]{2}{*}{ Dysithamnus mentalis (Temminck, 1823) } & 2 & 0 & 50,00 & Proterothrix s.1. sp. & 2 & 23 & 1 \\
\hline & & & 50,00 & Trouessartiidae & 0 & 0 & 2 \\
\hline Conopophagidae (1) & 7 & 1 & 100,00 & & 6 & & \\
\hline \multirow[t]{3}{*}{ Conopophaga lineata (Wied, 1831) } & 7 & 1 & 16,67 & Mesalgoides sp. & 0 & 1 & 0 \\
\hline & & & 16,67 & Neocalcealges sp. & 8 & 10 & 21 \\
\hline & & & 33,33 & Proctophyllodinae & 0 & 2 & 0 \\
\hline
\end{tabular}


Tabela 1. Continuação...

\begin{tabular}{|c|c|c|c|c|c|c|c|}
\hline Espécies de aves & $\mathbf{N}$ & Rc & $\operatorname{Prv}(\%)$ & Ácaros plumícolas & 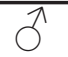 & 9 & Imaturos \\
\hline & & & 83,33 & Pterodectes sp. & 23 & 80 & 7 \\
\hline & & & 16,67 & Proterothrix s.l. sp. & 2 & 23 & 1 \\
\hline & & & 16,67 & Trouessartia incisa & 3 & 1 & 0 \\
\hline Dendrocolaptidae (3) & 12 & 2 & 50,00 & & & & \\
\hline Sittasomus griseicapillus (Vieillot, 1818) & 7 & 2 & 80,00 & Platyacarus sp. & 17 & 49 & 12 \\
\hline Dendrocolaptes platyrostris (Spix, 1825) & 2 & 0 & - & - & - & - & - \\
\hline Lepidocolaptes angustirostris (Vieillot, 1818) & 3 & 0 & 33,33 & Pterodectes sp. & 9 & 30 & 4 \\
\hline Furnariidae (6) & 18 & 2 & 56,25 & & & & \\
\hline Synallaxis frontalis (Pelzeln, 1859) & 1 & 0 & - & - & - & - & - \\
\hline \multirow[t]{3}{*}{ Synallaxis albescens (Temminck, 1823) } & 4 & 1 & 33,33 & Analges sp. & 1 & 6 & 7 \\
\hline & & & 33,33 & Proctophyllodinae & 0 & 1 & 0 \\
\hline & & & 66,67 & Pterodectes sp. & 2 & 5 & 2 \\
\hline \multirow[t]{4}{*}{ Synallaxis scutata (Sclater, 1859) } & 7 & 1 & 33,33 & Analgidae & 0 & 0 & 5 \\
\hline & & & 16,67 & Nycteridocaulus tyranni & 1 & 13 & 1 \\
\hline & & & 33,33 & Pterodectes sp. & 3 & 10 & 1 \\
\hline & & & 83,33 & Trouessartia incisa & 16 & 24 & 30 \\
\hline \multirow[t]{2}{*}{ Hylocryptus rectirostris (Wied, 1831) } & 2 & 0 & 50,00 & Analges sp. & 1 & 1 & 0 \\
\hline & & & 50,00 & Trouessartia capensis & 15 & 12 & 6 \\
\hline Lochmias nematura (Lichtenstein, 1823) & 2 & 0 & - & - & - & - & - \\
\hline Xenops rutilans (Temminck, 1821) & 2 & 0 & - & - & - & - & - \\
\hline Tyrannidae (14) & 146 & 10 & 42,96 & & & & \\
\hline \multicolumn{8}{|l|}{ Pipromorphinae } \\
\hline \multirow[t]{4}{*}{ Mionectes rufiventris (Cabanis, 1846) } & 9 & 0 & 11,11 & Anisodiscus sp. & 1 & 2 & 0 \\
\hline & & & 11,11 & Anisophyllodes sp. & 1 & 5 & 0 \\
\hline & & & 22,22 & Nycteriodocaulus tyranni & 1 & 16 & 0 \\
\hline & & & 22,22 & Pterodectinae & 0 & 5 & 4 \\
\hline \multirow[t]{2}{*}{ Leptopogon amaurocephalus (Tschudi, 1846) } & 9 & 0 & 33,33 & Nycteridocaulus tyranni & 11 & 16 & 0 \\
\hline & & & 22,22 & Trouessartia capensis & 1 & 4 & 7 \\
\hline Corythopis delalandi (Lesson, 1830) & 2 & 0 & 100,00 & Nycteridiocaulus lamellus & 4 & 16 & 1 \\
\hline \multicolumn{8}{|l|}{ Elaeniinae } \\
\hline \multirow[t]{2}{*}{ Elaenia flavogaster (Thunberg, 1822) } & 6 & 0 & 16,67 & Proctophyllodinae & 0 & 1 & 0 \\
\hline & & & 50,00 & Pterodectes sp. & 20 & 38 & 15 \\
\hline \multirow[t]{4}{*}{ Elaenia cristata (Pelzeln, 1868) } & 44 & 5 & 2,94 & Dolichodectes sp. & 1 & 0 & 0 \\
\hline & & & 2,94 & Nycteridocaulus tyranni & 1 & 0 & 0 \\
\hline & & & 23,53 & Pterodectes sp. & 18 & 32 & 53 \\
\hline & & & 5,88 & Trouessartia capensis & 1 & 1 & 0 \\
\hline \multirow[t]{4}{*}{ Elaenia chiriquensis (Lawrence, 1865) } & 43 & 2 & 7,32 & Anisophyllodes sp. & 4 & 8 & 0 \\
\hline & & & 2,44 & Dolichodectes neotropicus & 6 & 14 & 33 \\
\hline & & & 29,27 & Pterodectes sp. & 141 & 162 & 325 \\
\hline & & & 7,32 & Trouessartia capensis & 3 & 4 & 8 \\
\hline Camptostoma obsoletum (Temminck, 1824) & 8 & 2 & 83,33 & Pterodectes sp. & 46 & 64 & 128 \\
\hline \multirow[t]{2}{*}{ Suiriri suiriri (Vieillot, 1818) } & 2 & 0 & 50,00 & Proctophyllodinae & 1 & 0 & 0 \\
\hline & & & 50,00 & Pterodectes sp. & 29 & 36 & 43 \\
\hline Phylloscartes sp. & 1 & 0 & - & - & - & - & - \\
\hline Culicivora caudacuta (Vieillot, 1818) & 3 & 0 & - & - & - & - & - \\
\hline \multirow[t]{2}{*}{ Tolmomyias sulphurescens (Spix, 1825) } & 6 & 0 & 50,00 & Nycteridiocaulus tyranni & 1 & 13 & 0 \\
\hline & & & 16,67 & Trouessartia sp. & 2 & 3 & 1 \\
\hline Platyrinchus mystaceus (Vieillot, 1818) & 1 & 0 & 100,00 & Proctophyllodinae & 0 & 3 & 0 \\
\hline \multicolumn{8}{|l|}{ Fluvicolinae } \\
\hline \multirow[t]{2}{*}{ Lathrotriccus euleri (Cabanis, 1868) } & 11 & 1 & 20,00 & Proctophyllodinae & 0 & 11 & 0 \\
\hline & & & 30,00 & Pterodectes sp. & 26 & 65 & 39 \\
\hline
\end{tabular}


Tabela 1. Continuação...

\begin{tabular}{|c|c|c|c|c|c|c|c|}
\hline Espécies de aves & $\mathbf{N}$ & Rc & $\operatorname{Prv}(\%)$ & Ácaros plumícolas & $\pi$ & 9 & Imaturos \\
\hline Knipolegus lophotes Boie, 1828 & 1 & 0 & 100,00 & Trouessartiidae & 0 & 0 & 4 \\
\hline Pipridae (1) & 56 & 4 & 44,23 & & & & \\
\hline \multirow[t]{5}{*}{ Antilophia galeata (Lichtenstein, 1823) } & 56 & 4 & 1,92 & Diproctophyllodes sp. & 1 & 13 & 0 \\
\hline & & & 1,92 & Mimicalges sp. & 1 & 9 & 0 \\
\hline & & & 38,46 & Proctophyllodinae & 0 & 98 & 2 \\
\hline & & & 7,69 & Pterodectinae & 0 & 4 & 0 \\
\hline & & & 3,85 & Trouessartia sp. & 2 & 4 & 0 \\
\hline Tityridae (1) & 8 & 4 & 100,00 & & & & \\
\hline \multirow[t]{3}{*}{ Schiffornis virescens (Lafresnaye, 1838) } & 8 & 4 & 50,00 & Nycteriodocaulus tyranni & 44 & 75 & 18 \\
\hline & & & 50,00 & Nycteridiocaulus pectinatus & 3 & 34 & 0 \\
\hline & & & 25,00 & Pterodectinae & 0 & 1 & 0 \\
\hline Vireonidae (1) & 3 & 0 & - & & & & \\
\hline Cyclarhis gujanensis (Gmelin, 1789) & 3 & 0 & - & - & - & - & - \\
\hline Hirundinidae (2) & 5 & 0 & 40,00 & & 2 & & \\
\hline \multirow[t]{2}{*}{ Alopochelidon fucata (Temminck, 1822) } & 2 & 0 & 100,00 & Scutulanyssus sp. & 8 & 8 & 1 \\
\hline & & & 100,00 & Trouessartia sp. & 21 & 25 & 0 \\
\hline Stelgidopteryx ruficollis (Vieillot, 1817) & 3 & 0 & - & - & - & - & - \\
\hline Troglodytidae (3) & 27 & 3 & 4,17 & & & & \\
\hline Cistothorus platensis (Latham, 1790) & 1 & 0 & - & - & - & - & - \\
\hline Troglodytes musculus (Naumann, 1823) & 6 & 0 & 16,67 & Trouessartiidae & 0 & 0 & 17 \\
\hline Thryothorus leucotis (Lafresnaye, 1845) & 20 & 3 & - & - & - & - & - \\
\hline Turdidae (5) & 27 & 3 & 75,00 & & & & \\
\hline \multirow[t]{2}{*}{ Catharus fuscescens (Stephens, 1817) } & 1 & 0 & 100,00 & Proctophyllodinae & 0 & 2 & 0 \\
\hline & & & 100,00 & Pterodectes sp. & 3 & 13 & 8 \\
\hline \multirow[t]{3}{*}{ Turdus rufiventris (Vieillot, 1818) } & 7 & 0 & 14,29 & Analges sp. & 1 & 0 & 0 \\
\hline & & & 57,14 & Pterodectes sp. & 11 & 18 & 6 \\
\hline & & & 14,29 & Trouessartia sp. & 4 & 6 & 0 \\
\hline \multirow[t]{3}{*}{ Turdus leucomelas (Vieillot, 1818) } & 15 & 3 & 33,33 & Proctophyllodinae & 0 & 5 & 0 \\
\hline & & & 50,00 & Pterodectes sp. & 65 & 76 & 42 \\
\hline & & & 66,67 & Trouessartia incisa & 16 & 37 & 27 \\
\hline Turdus amaurochalinus (Cabanis, 1850) & 2 & 0 & - & - & - & - & - \\
\hline Turdus albicollis (Vieillot, 1818) & 2 & 0 & 100,00 & Pterodectes sp. & 20 & 43 & 35 \\
\hline Mimidae (1) & 5 & 2 & 100,00 & & & & \\
\hline Mimus saturninus (Lichtenstein, 1823) & 5 & 2 & 100,00 & Pterodectes sp. & 24 & 49 & 65 \\
\hline Coerebidae (1) & 2 & 0 & - & & & & \\
\hline Coereba flaveola (Linnaeus, 1758) & $\mathrm{x} 2$ & 0 & - & - & - & - & - \\
\hline Thraupidae (10) & 78 & 9 & 44,93 & & & & \\
\hline Schistochlamys melanopis (Latham, 1790) & 1 & 0 & 100,00 & Proctophyllodes sp. & 2 & 0 & 2 \\
\hline \multirow[t]{3}{*}{ Neothraupis fasciata (Lichtenstein, 1823) } & 23 & 1 & 4,55 & Analges sp. & 0 & 1 & 0 \\
\hline & & & 9,09 & Pterodectes sp. & 2 & 9 & 4 \\
\hline & & & 4,55 & Trouessartia sp. & 6 & 6 & 1 \\
\hline Pyrrhocoma ruficeps (Strickland, 1844) & 3 & 0 & - & - & - & - & - \\
\hline Cypsnagra hirundinacea (Lesson, 1831) & 4 & 0 & - & - & - & - & - \\
\hline \multirow[t]{4}{*}{ Trichothraupis melanops (Vieillot, 1818) } & 26 & 7 & 10,53 & Atelanalges sp. & 0 & 1 & 5 \\
\hline & & & 10,53 & Proctophyllodinae & 0 & 2 & 1 \\
\hline & & & 68,42 & Pterodectes sp. & 118 & 233 & 122 \\
\hline & & & 21,05 & Trouessartia capensis & 21 & 20 & 0 \\
\hline \multirow[t]{2}{*}{ Eucometis penicillata (Spix, 1825) } & 8 & 1 & 71,43 & Pterodectes sp. & 12 & 22 & 23 \\
\hline & & & 28,57 & Trouessartia capensis & 43 & 44 & 24 \\
\hline Tachyphonus rufus (Boddaert, 1783) & 4 & 0 & 100,00 & Pterodectes sp. & 46 & 50 & 84 \\
\hline
\end{tabular}


Tabela 1. Continuação...

\begin{tabular}{|c|c|c|c|c|c|c|c|}
\hline Espécies de aves & $\mathbf{N}$ & $\mathbf{R c}$ & $\operatorname{Prv}(\%)$ & Ácaros plumícolas & $\hat{0}$ & 9 & Imaturos \\
\hline & & & 25,00 & Trouessartiidae & 0 & 0 & 12 \\
\hline \multirow[t]{2}{*}{ Thraupis sayaca (Linnaeus, 1766) } & 3 & 0 & 33,33 & Proctophyllodinae & 0 & 4 & 0 \\
\hline & & & 33,33 & Trouessartia sp. & 3 & 3 & 0 \\
\hline Thraupis palmarum (Wied, 1823) & 1 & 0 & - & - & - & - & - \\
\hline Tangara cayana (Linnaeus, 1766) & 5 & 0 & 20,00 & Pterodectes sp. & 14 & 18 & 14 \\
\hline Emberizidae (9) & 164 & 22 & 37,86 & & & & \\
\hline \multirow[t]{5}{*}{ Zonotrichia capensis (Statius Muller, 1776) } & 29 & 3 & 3,85 & Analges sp. & 0 & 1 & 2 \\
\hline & & & 3,85 & Hadrophyllodes sp. & 5 & 36 & 6 \\
\hline & & & 3,85 & Mesalgoides sp. & 1 & 1 & 5 \\
\hline & & & 20,83 & Pterodectes sp. & 10 & 22 & 35 \\
\hline & & & 46,15 & Trouessartia capensis & 11 & 34 & 132 \\
\hline \multirow[t]{4}{*}{ Ammodramus humeralis (Bosc, 1792) } & 38 & 7 & 9,68 & Analges sp. & 3 & 0 & 6 \\
\hline & & & 3,23 & Proctophyllodes macedo & 3 & 4 & 0 \\
\hline & & & 16,13 & Pterodectes sp. & 25 & 40 & 47 \\
\hline & & & 48,39 & Trouessartia sp. & 57 & 131 & 76 \\
\hline \multirow[t]{3}{*}{ Arremon flavirostris (Swainson, 1838) } & 13 & 3 & 10,00 & Nycteriodocaulus tyranni & 2 & 0 & 0 \\
\hline & & & 80,00 & Pterodectes sp. & 68 & 118 & 50 \\
\hline & & & 30,00 & Trouessartia sp. & 1 & 0 & 5 \\
\hline \multirow[t]{2}{*}{ Sicalis citrina (Pelzeln, 1870) } & 2 & 0 & 50,00 & Pterodectes sp. & 3 & 0 & 0 \\
\hline & & & 50,00 & Trouessartia incisa & 9 & 2 & 2 \\
\hline Emberizoides herbicola (Vieillot, 1817) & 31 & 4 & - & - & - & - & - \\
\hline \multirow[t]{3}{*}{ Volatinia jacarina (Linnaeus, 1766) } & 31 & 5 & 3,85 & Mesalgoides sp. & 1 & 0 & 2 \\
\hline & & & 15,38 & Pterodectes sp. & 4 & 9 & 47 \\
\hline & & & 30,77 & Trouessartia sp. & 58 & 55 & 10 \\
\hline \multirow[t]{2}{*}{ Sporophila sp. } & 16 & 0 & 6,25 & Proctophyllodes sporophilae & 1 & 1 & 1 \\
\hline & & & 6,25 & Trouessartia sp. & 2 & 8 & 5 \\
\hline Sporophila plumbea (Wied, 1830) & 2 & 0 & - & - & - & - & - \\
\hline Sporophila nigricollis (Vieillot, 1823) & 2 & 0 & - & - & - & - & - \\
\hline Cardinalidae (3) & 23 & 4 & 100,00 & & & & \\
\hline \multirow[t]{2}{*}{ Saltator maximus (Statius Muller, 1776) } & 5 & 0 & 100,00 & Pterodectes sp. & 83 & 103 & 145 \\
\hline & & & 20,00 & Trouessartia incisa & 1 & 1 & 1 \\
\hline Saltator coerulescens (Vieillot, 1817) & 2 & 0 & - & - & - & - & - \\
\hline \multirow[t]{2}{*}{ Saltator similis (d’Orbigny \& Lafresnaye, 1837) } & 16 & 4 & 75,00 & Pterodectes sp. & 165 & 192 & 388 \\
\hline & & & 25,00 & Trouessartia incisa & 9 & 11 & 20 \\
\hline Parulidae (4) & 37 & 7 & 66,67 & & & & \\
\hline Geothlypis aequinoctialis (Gmelin, 1789) & 2 & 0 & 50,00 & Pterodectinae & 0 & 1 & 0 \\
\hline \multirow[t]{4}{*}{ Basileuterus hypoleucus (Bonaparte, 1830) } & 16 & 1 & 6,67 & Pandalurinae & 1 & 0 & 1 \\
\hline & & & 13,33 & Proctophyllodinae & 0 & 4 & 0 \\
\hline & & & 53,33 & Pterodectes sp. & 38 & 76 & 78 \\
\hline & & & 26,67 & Trouessartia sp. & 24 & 22 & 1 \\
\hline \multirow[t]{5}{*}{ Basileuterus flaveolus (Baird, 1865) } & 19 & 6 & 9,09 & Analges sp. & 0 & 1 & 0 \\
\hline & & & 9,09 & Calcealges novimundus & 2 & 0 & 0 \\
\hline & & & 27,27 & Nycteridocaulus tyranni & 1 & 6 & 5 \\
\hline & & & 76,92 & Pterodectes sp. & 49 & 141 & 111 \\
\hline & & & 69,23 & Trouessartia sp. & 81 & 58 & 51 \\
\hline Total & 696 & 80 & 44,98 & - & 1710 & 3049 & 2525 \\
\hline
\end{tabular}

$\mathrm{N}$ = Número de indivíduos capturados; $\mathrm{Rc}=$ Número de indivíduos recapturados; Prv = Prevalência; * Contaminação 


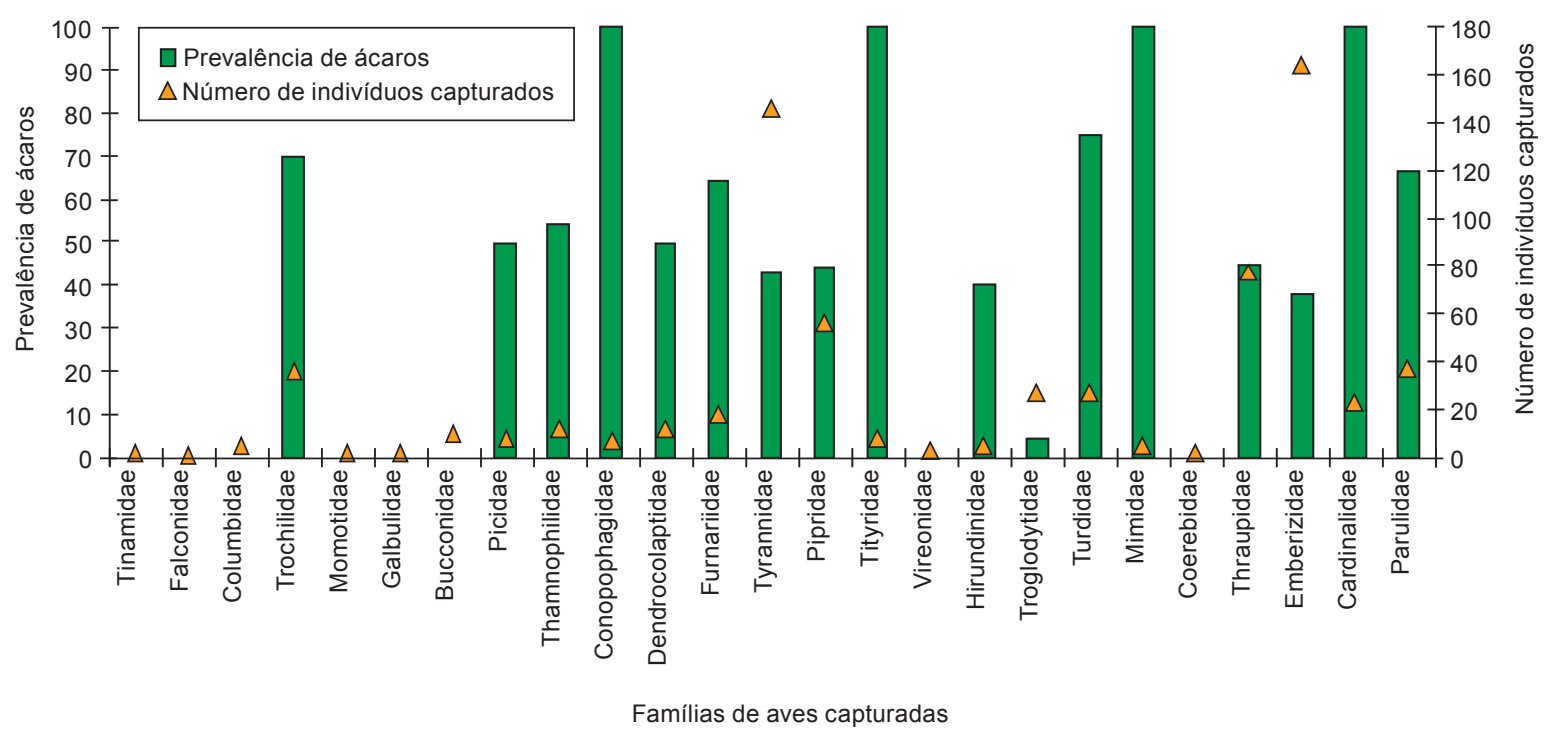

Figura 1. Prevalência de ácaros plumícolas e número de aves capturadas por famílias de aves capturadas no Cerrado da Fazenda Água Limpa, Brasília, Distrito Federal, Brasil, durante o período de janeiro a agosto de 2002.

Figure 1. Prevalence of feather mites and the compared with number of birds collected separated by bird families in Cerrado of Fazenda Água Limpa, Brasília, Distrito Federal, Brazil, between January and August, 2002.

pelo grande número de indivíduos capturados. Algumas famílias de Passeriformes, como Vireonidae e Coerebidae, não apresentaram ácaros plumícolas talvez pelo baixo número de indivíduos amostrados (Figura 1).

Na família de ácaros Avenzoariidae, o gênero Pterotrogus foi encontrado apenas em Venilliornis passerinus e Scutylanyssus em Alopochelidon fucata, o que concorda com a distribuição deste gênero entre os Piciformes e Apodiformes, respectivamente (Gaud \& Atyeo 1996). Enquanto na família Psoroptoididae houve dois registros de espécimes identificados apenas até subfamília, Pandarulinae, em Picoides mixtus e Basileuterus hypoleucus. Ainda entre os Psoroptoididae, dois registros do gênero Mesalgoides em Conopophaga lineata e Volatinia jacarina.

Há registros de ocorrência da família Psoroptoididae em nove ordens de aves, sendo o gênero Mesalgoides freqüente em Apodiformes, Passeriformes e Piciformes (Gaud \& Atyeo 1996). Este gênero esteve presente em dois indivíduos de Passeriformes, nas famílias Conopophagidae e Emberizidae. No Brasil, Lyra-Neves et al. (2000) encontraram o gênero Mesalgoides sp. em três espécies de Thraupidae e uma de Emberizidae. Rojas (1998) e Roda \& Farias (1999) encontraram Mesalgoides sp. também em Volatinia jacarina (Emberizidae). Entretanto, Lyra-Neves et al. (2003) encontraram este mesmo gênero nas famílias Thraupidae e Coerebidae como ocorrência ocasional.

Os indivíduos da família Trouessartiidae, que apresentaram prevalência igual ou superior a $50 \%$, estiveram presentes em aves das famílias Furnariidae, Hirundinidae, Turdidae, Emberizidae, Parulidae e Thamnophilidae (Tabela 1). Esta família de ácaros plumícolas apresentou prevalência de 17,21\% (ocorrendo em 34 espécies e 18 famílias de aves), com o gênero Trouessartia presente em 96 indivíduos (15,58\% das aves com este gênero de ácaro plumícola, pertencentes 25 espécies e 10 famílias), tanto nesta família como no gênero citado apresentaram-se como segundo mais prevalentes no material analisado (Tabela 2).

O gênero Trouessartia, ainda na família Trouessartidae, é encontrado em aves pertencentes a 10 famílias de Passeriformes (Santana 1976, Gaud \& Atyeo 1996), no Brasil apresenta ampla ocorrência entre aves de várias regiões do país (Rojas 1998, Roda \& Farias 1999, Lyra-Neves et al. 2000, 2003). Neste estudo foram encontrados 96 indivíduos $(15,58 \%)$ com ocorrência deste gênero de ácaro, das quais duas espécies foram identificadas especificamente: Trouessartia incisa (Conopophagidae, Furnariidae, Turdidae, Emberizidae e Cardinalidae) e T. capensis (Furnariidae, Tyrannidae, Thraupidae e Emberizidae). Até então, $T$. incisa havia sido associada apenas a aves da família Turdidae (Santana 1976) e T. capensis apenas em seu hospedeiro tipo, Zonotrichia capensis (Emberizidae) (Santana 1976).

Nos não Passeriformes a ocorrência de Trouessartidae esteve relacionada aos Picidae, com o gênero Pseudalges sp., que é comumente encontrado em aves da família Meropidae (Coraciiformes) (Orwig 1968). Espécimes de Neocalcealges sp. foram encontrados em Trochilidae, o que parece ser um encontro acidental a julgar pelos hospedeiros habituais das espécies encontradas nesta família, uma vez que as seis espécies deste gênero são encontradas em Tinamidae e Turdidae (Orwig 1968, Gaud \& Atyeo, 1996). Por outro lado sua ocorrência foi considerada aceitável na única espécie de ave capturada da família Conopophagidae (Tabela 1). O gênero Calcealges ocorre em sete famílias de Passeriformes (Orwig 1968), uma espécie identificada, C. novimundus Orwig 1968, foi encontrada em Thamnophilus punctatus, T. caerulescens e Basileuterus flaveolus (Thamnophilidae e Parulidae, respectivamente), elevando a nove o número de famílias em que se encontra este gênero. Segundo Orwig (1968), esta espécie de ácaro até então havia sido encontrada apenas em Thamnophilus doliatus (Linnaeus, 1764).

Indivíduos da família Proctophyllodidae (com prevalência $\geq 50 \%$ ) estiveram presentes em espécies das famílias Trochilidae, Picidae, Thamnophilidae, Conopophagidae, Dendrocolaptidae, Furnariidae, Tyrannidae, Tityridae, Turdidae, Mimidae, Thraupidae, Emberizidae, Cardinalidae e Parulidae (Tabela 1). Esta família de ácaros foi a mais prevalente (30\%), encontrada em 183 indivíduos de aves, presentes em 49 espécies e 15 famílias, sendo Pterodectes o gênero mais freqüente nas aves analisadas e com maior prevalência 19,64\%,(121 indivíduos de 30 espécies/11 famílias), seguido por Nycteridocaulus (22 indivíduos, 9/5) e Proctophyllodes (18 indivíduos 6/4) (Tabela 2). 
Tabela 2. Freqüência absoluta e prevalência dos gêneros de ácaros plumícolas nas aves capturadas na Fazenda Água Limpa, Brasília, Distrito Federal, Brasil, durante o período de janeiro a agosto de 2002.

Table 2. Absolute frequency and prevalence of feather mites genus on of birds collected separated by bird families of Fazenda Água Limpa, Brasília, Distrito Federal, Brazil, between January and August, 2002.

\begin{tabular}{|c|c|c|}
\hline & Frq & $\operatorname{Prv}(\%)$ \\
\hline \multicolumn{3}{|l|}{ ANALGOIDEA } \\
\hline \multicolumn{3}{|l|}{ ANALGIDAE } \\
\hline \multicolumn{3}{|l|}{ Analginae } \\
\hline Analges & 11 & 1,79 \\
\hline Atelanalges & 2 & 0,32 \\
\hline \multicolumn{3}{|l|}{ AVENZOARIIDAE } \\
\hline \multicolumn{3}{|l|}{ Pteronyssinae } \\
\hline Pterotrogus & 1 & 0,16 \\
\hline Scutulanyssus & 2 & 0,32 \\
\hline \multicolumn{3}{|l|}{ TROUESSARTIIDAE } \\
\hline Trouessartia & 96 & 15,58 \\
\hline Calcealges & 6 & 0,97 \\
\hline Neocalcealges & 2 & 0,32 \\
\hline Pseudalges & 1 & 0,16 \\
\hline \multicolumn{3}{|l|}{ PSOROPTOIDIDAE } \\
\hline \multicolumn{3}{|l|}{ Pandalurinae } \\
\hline Mesalgoides & 3 & 0,49 \\
\hline \multicolumn{3}{|c|}{ PROCTOPHYLLODIDAE } \\
\hline \multicolumn{3}{|c|}{ Proctophyllodinae } \\
\hline Proctophyllodes & 18 & 2,92 \\
\hline Nycteridocaulus & 22 & 3,57 \\
\hline Mimicalges & 1 & 0,16 \\
\hline Anisophyllodes & 2 & 0,32 \\
\hline Diproctophyllodes & 1 & 0,16 \\
\hline Hadrophyllodes & 1 & 0,16 \\
\hline Proterothrix s.l. & 2 & 0,32 \\
\hline Platyacarus sp. & 5 & 0,81 \\
\hline \multicolumn{3}{|l|}{ Pterodectinae } \\
\hline Pterodectes & 121 & 19,64 \\
\hline Anisodiscus & 1 & 0,16 \\
\hline Dolichodectes & 2 & 0,32 \\
\hline Toxerodectes & 9 & 1,46 \\
\hline \multicolumn{3}{|l|}{ Ramphocaulinae } \\
\hline Allodectes & 3 & 0,49 \\
\hline
\end{tabular}

Frq = Frequência absoluta; Prv = Prevalência

A família Proctophyllodidae, encontrada primariamente em famílias de Passeriformes (Gaud \& Atyeo 1996), foi neste estudo também registrada em Trochilidae (20 indivíduos) e Picidae (um indivíduo), como já foi relatado por Park \& Atyeo (1971b). Foram identificados 13 gêneros pertencentes às três subfamílias de Proctophyllodidae (Proctophyllodinae, Pterodectinae e Rhamphocaulinae) (Tabela 2), dos quais Pterodectes foi o gênero mais prevalente. Outros estudos também constataram a alta prevalência deste gênero em Passeriformes do cerrado e mata de galeria em Minas Gerais (Rojas 1998), na Zona da Mata (Roda \& Farias 1999) e da Mata Atlântica em Pernambuco (Lyra-Neves et al. 2003). Essa grande prevalência parece ser inversamente proporcional ao número de espécies atualmente conhecidas (Park \& Atyeo 1971b, Hernandes \& Valim 2005, 2006, OConnor et al. 2005, Valim \& Hernandes 2006).

Atyeo \& Braasch (1966) indicam que 80\% das espécies conhecidas do gênero Proctophyllodes são específicas de Passeriformes, entretanto Proctophyllodes huitzilopochtlii é restrito a família Trochilidae, como observado para as aves da região de Cerrado, Distrito Federal, Brasília. Embora esta espécie seja encontrada em 13 espécies de Trochilidae (Atyeo \& Braasch 1966) as duas espécies de beija-flores encontradas associadas a este ácaro (Amazilia fimbriata e Chlorostilbon aureoventris) são pela primeira vez reportadas. Apenas duas espécies foram identificadas em aves da ordem Passeriformes: Proctophyllodes macedo (em Ammodramus humeralis) que ocorre em duas espécies de Motacillidae, sem registro para região neotropical; e P. sporophilae (em Sporophila sp.) encontrado até então em duas espécies de Sporophila no México (Atyeo \& Braasch 1966). Espécimes do gênero Proctophyllodes ocorreram apenas em aves das famílias Thraupidae. Alguns trabalhos têm mostrado que este gênero ocorre no Brasil nas famílias Turdidae, Thraupidae, Fringillidae, Coerebidae, Furnariidae, Emberizidae e Tyrannidae (Rojas 1998, Roda \& Farias 1999, Lyra-Neves et al. 2003), entretanto ele é encontrado em 20 famílias de Passeriformes em todo mundo (Atyeo \& Braasch 1966).

Dentre outros gêneros de Proctophyllodinae encontrados, o segundo mais prevalente foi Nycteridocaulus (Tabela 2). Das sete espécies encontradas neste gênero (Atyeo 1966, Alzuet \& Brandetti 1986) três delas foram coletadas em Brasília. A primeira, Nycteridocaulus lamellus, até então associada com Myarchus crinitus e Tyrannus tyrannus (ambos Tyrannidae) nos EUA, foi encontrada em Corythopis delalandi (Tyrannidae) em Brasília; e duas outras espécies, Nycteridocaulus tyranni e Nycteridocaulus pectinatus sempre associadas com Schiffornis virescens (Tityridae). Dado a distribuição destas espécies de Nycteridocaulus (Atyeo 1966, Alzuet \& Brandetti 1986), esses achados de hospedeiros trazem registros novos.

As espécies de Pterodectinae identificadas foram Toxerodectes subulatus (em Colibri serrirostris), espécie comumente encontrada nesta espécie de ave e já registrada nos estados de Mato Grosso, São Paulo e Minas Gerais no Brasil (Park \& Atyeo 1974) e T. biscutatus (em Thalurania furcata), que é conhecido apenas por sua associação com a espécie Colibri delphinae (em Honduras, Peru e Venezuela) (Park \& Atyeo 1975). Estes mesmos autores encontraram na Guatemala espécimes em T. furcata de Toxerodectes do 'complexo hastifolia', mesmo grupo de espécies de T. biscutatus. O achado de um Pterodectes sp. em Thalurania furcata é provavelmente resultado de contaminação, pois é sabido que as espécies deste gênero não ocorrem em aves da família Trochilidae (Park \& Atyeo 1971b). Um membro da subfamília Pterodectinae foi identificado como Proterothrix sensu lato (em Dysithamnus mentalis e Conopophaga lineata) visto que através da chave dicotômica proposta por Gaud \& Atyeo (1996) é possível classificar os espécimes a este gênero, entretanto suas características genéricas não são concordantes com as descritas por Park \& Atyeo (1971b).

Uma subfamília que é encontrada exclusivamente nos Trochilidae é Rhamphocaulinae, onde vivem como siringícolas nas penas de seus hospedeiros (Gaud \& Atyeo 1996). Uma única espécie foi encontrada, Allodectes amadoi em Colibri serrirostris e Thalurania furcata. Esta espécie havia sido encontrada apenas em duas outras espécies de Colibri spp. no México e no Equador (Park \& Atyeo 1971a).

Poulin (1991) considera que a prevalência quando baixa ou nula pode indicar falta de exposição aos ectoparasitos e não a baixa susceptibilidade, o valor observado nas aves do Cerrado de Brasília $(44,98 \%)$ esteve de acordo com a média dos resultados obtidos em outros trabalhos realizados no Brasil (Marini et al. 1996, Marini \& Couto 1997, Rojas 1998, Lyra-Neves et al. 2000, 2003) em que foram observadas variações de 28,7 a $97,9 \%$ em seus estudos. Como se pôde observar os ácaros plumícolas formam um grande grupo de artrópodes associados às aves da fauna Brasileira, embora muitas espécies ainda permaneçam desconhecidas para a ciência, os estudos faunísticos são fundamentais para contribuição ao conhecimento da biodiversidade destes ácaros ainda pouco estudados em um país com uma das maiores avifaunas do mundo. 


\section{Agradecimentos}

À CAPES pelo fornecimento da bolsa de estudo à autora sênior (MFK) que juntamente com a UnB - Universidade de Brasília permitiu a realização da pesquisa. $\mathrm{O}$ autor júnior (MAM) recebe bolsa de pesquisador do CNPq. À administração da Fazenda Água Limpa que possibilitou a coleta de dados. Agradecemos ao CEMAVE/IBAMA pelo fornecimento das anilhas de metal. Fabio A. Hernandes (Departamento de Zoologia e Botânica. Universidade Estadual Paulista - UNESP) e Wallace R. Telino Júnior (Universidade Federal Rural de Pernambuco) pela revisão crítica do manuscrito.

\section{Referências Bibliográficas}

AB'SABER, A.N. 1983. O domínio dos Cerrados: introdução ao conhecimento. Rev. Serv. Publ. 111:41-55.

ALZUET, A.B.D. \& BRANDETTI, E. 1986. Ácaros plumícolas, ectoparásitos de aves silvestres (Astigmata: Proctophyllodidae). Neotropica 32(87):81-87.

ATYEO, W.T. 1966. A new genus and six new species of feather mites primarily from Tyranni (Acarina: Proctophyllodidae). J. Kans. Entomol. Soc. 39:481-492.

ATYEO, W.T. \& BRAASCH, N.L. 1966. The feather mite genus Proctophyllodes (Sarcoptiformes: Proctophyllodidae). Bull. Univ. Nebr. State Mus. 5:1-354.

BLANCO, G., TELLA, J.L., POTTI, J. \& BRAZ, A. 2001. Feather mites on birds: costs of parasitism or conditional outcomes? J. Avian Biol. 32:271-274.

BUSH, A.O., LAFFERTY, K.D., LOTZ, J.M. \& SHOSTAK, A.W. 1997. Parasitology meets ecology on its own terms: Margolis et al. revisited. J. Parasitol. 83:575-83.

CARVALHO, A.R. \& SERRA-FREIRE, N.M. 2001. Identificação de ácaros Astigmata encontrados em penas de pipira-do-papo-vermelho (Ramphocelus carbo) em Belém, Pará, Brasil. Tangara 1(2):85-87.

COMITÊ BRASILEIRO DE REGISTROS ORNITOLÓGICOS. 2007. Listas das aves do Brasil. Versão 16/08/2007 Disponível em http://www.ib.usp. br/cbro (Acesso em: 16/08/2007).

EITEN, G. 1972. The cerrado vegetation of Brazil. Bot. Rev. 38:201-341.

FIGUEROLA, J. 2000. Ecological correlates of feather mite prevalence in passerines. J. Avian Biol. 31:489-494.

FLECHTMANN, C.H.W. 1975. Elementos de Acarologia. Livraria Nobel S.A., São Paulo, 344p.

GAUD, J. \& ATYEO, W.T. 1976. Discordances entre les aires de. répartition géographique des parasites et celles de leurs hôtes. Acarologia 18:329-344.

GAUD, J. \& ATYEO, W.T. 1996. Feather mites of the world (Acarina, Astigmata): the supraspecific taxa. (Part. I). Ann. Mus. R. Afr. Cent. Sci. Zool. 277:1-187.

HERNANDES, F.A. \& VALIM, M.P. 2005. A new species of Pterodectes Robin, 1877 (Proctophyllodidae: Pterodectinae) from the pale-breasted thrush, Turdus leucomelas (Passeriformes: Turdidae). Zootaxa 1081:61-68.

HERNANDES, F.A. \& VALIM, M.P. 2006. Two new species of the feather mite subfamily Pterodectinae (Acari: Astigmata: Proctophyllodidae) from Brazil. Zootaxa 1235:49-61.

KLINK, C.A. \& MACHADO, R.B. 2005. Conservation of the Brazilian Cerrado. Conserv. Biol. 19:707-713.

LYRA-NEVES, R.L., FARIAS, A.M.I., JÚNIOR, W.R.T., BOTELHO, M.C.N., LIMA, M.C.A. 2000. Ectoparasitismo em aves silvestres (Passeriformes- Emberizidae) de Mata Atlântica, Igarassu, Pernambuco. Melopsittacus 3(2):64-71.

LYRA-NEVES, R.M., ISIDRO-DE-FARIAS, Â.M. \& TELINO-JÚNIOR, W.R. 2003. Ecological relationships between feather mites (Acari) and wild birds of Emberizidae (Aves) in a fragment of Atlantic Forest in northeastern Brazil. Rev. Bras. Zool. 20(3):481-485.
MARINI, M.Â. \& COUTO, D. 1997. Correlações ecológicas entre ectoparasitos e aves de floresta de Minas Gerais. In Contribuição ao Conhecimento Ecológico do Cerrado (L.L. Leite \& C.H. Saito, eds.). Departamento de Ecologia, Universidade de Brasília, p. 210-218.

MARINI, M.Â., REINERT, B.L., BORNSCHEIN, M.R., PINTO, J.C. \& PICHORIM, M.A. 1996. Ecological correlates of ectoparasitism on Atlantic Forest birds, Brazil. Ararajuba 4(2):93-102.

MIRONOV, S.V. 2003. On some problems in systematics of feather mites. Acarina 11:3-29.

OCONNOR, B.M., FOUFOPOULOS, J., LIPTON, D. \& LINDSTRÖM, K. 2005. Mites associated with the small ground finch, Geospiza fuliginosa (Passeriformes: Emberizidae), from the Galápagos islands. J. Parasitol. 91:1304-1313.

ORWIG, K.R. 1968. The genera and species of the feather mite subfamily Trouessartinae, except Trouessartia (Acarina: Proctophyllodidae). Bull. Univ. Nebr. State Mus. 8(1):1-187.

PACHECO, J.F. \& PARRINI, R. 1999. A atividade naturalística de Herbert Franzoni Berla (1912-1985), ornitólogo e acarologista do Museu Nacional. Atual. Ornitol. 87:4-6.

PARK, C.K. \& ATYEO, W.T. 1971a. The species of a new subfamily of feather mites, the Allodectinae (Analgoidea: Proctophyllodidae). Redia 52:653-678.

PARK, C.K. \& ATYEO, W.T. 1971b. A generic revision of the Pterodectinae, a new subfamily of feather mites (Sarcoptiformes: Analgoidea). Bull. Univ. Nebr. State Mus. 9:39-88.

PARK, C.K. \& ATYEO, W.T. 1973. The pterodectine feather mites of hummingbirds: The genus Toxerodectes Park \& Atyeo (the hastifolia group). J. Ga. Entomol. Soc. 8:221-233.

PARK, C.K. \& ATYEO, W.T. 1974. The pterodectine feather mites of hummingbirds: The genus Toxerodectes Park \& Atyeo (the lecroyae and gladiger group). J. Ga. Entomol. Soc. 9:18-32.

PARK, C.K. \& ATYEO, W.T. 1975. The Pterodectine feather mites of hummingbirds: the genus Xynonodectes Park and Atyeo. J. Ga. Entomol. Soc. 10(22):128-144.

POULIN, R. 1991. Group-living and infestation by ectoparasites in Passerines. Condor 93:418-423.

PROCTOR, H.C. 2003. Feather mites (Acari: Astigmata): ecology, behavior and evolution. Annu. Rev. Entomol. 48:185-209.

PROCTOR, H. \& OWENS, I. 2000. Mites and birds: diversity, parasitism and coevolution. Trends Ecol. Evol. 15:358-364.

RATTER, J.A. 1980. Notes on the vegetation of Fazenda Água Limpa. (Brasília, DF, Brasil). Royal Botanical Garden, Edinburgh, 111p.

RODA, S.A. \& FARIAS, A.M.I. 1999. Ácaros plumícolas em aves Passeriformes da Zona da Mata Norte de Pernambuco, Brasil. Rev. Bras. Zool. 16:879-886.

ROJAS, M.R.R. 1998. Correlações ecológicas entre ectoparasitos e aves de floresta e cerrado nas áreas de proteção do Barreiro e Mutuca, Municípios de Belo Horizonte e Nova Lima, Minas Gerais. Dissertação de Mestrado. Curso de Pós-Graduação em Ecologia da Conservação e Manejo da Vida Silvestre. Instituto de Ciências Biológicas, Universidade Federal de Minas Gerais, 65p.

SANTANA, F.J. 1976. A review of the genus Trouessartia (Analgoidea: Alloptidae). J. Med. Entomol. Suppl. 1(1):1-128.

STORNI, A., ALVES, M.A.S. \& VALIM, M.P. 2005. Ácaros de penas e carrapatos (Acari) associados a Turdus albicollis Vieillot (Aves: Muscicapidae) em uma área de Mata Atlântica da Ilha Grande, Rio de Janeiro. Rev. Bras. Zool. 22(2):419-423.

VALIM, M.P. \& HERNANDES, F.A. 2006. Redescriptions of four species of the feather mite genus Pterodectes Robin, 1877 (Acari: Proctophyllodidae: Pterodectinae) described by Herbert F. Berla. Acarina, 14(1):41-55 